\title{
Intestinal Parasitic Infections in School Students in Malakal City, Upper Nile State, South Sudan
}

\author{
Magdi Bayoumi ${ }^{1}$, Opwonyi Nykwac ${ }^{1}$, Mohammed Kardaman' ${ }^{1}$ Mans Ullberg ${ }^{2}$, Eyad M. \\ Alshammari $^{3}$, Gunnar Sandström², Amir Saeed ${ }^{1,2,3}$, Hadi Abd ${ }^{2,3 *}$ \\ ${ }^{1}$ University of Medical Sciences and Technology, Faculty of Medical Laboratory Sciences, Department of Parasitology, Khartoum, Sudan \\ ${ }^{2}$ Karolinska Institute, Department of Laboratory Medicine, Division of Clinical Microbiology F 82 and Karolinska University Hospital, Huddinge, SE-141 86 \\ Stockholm, Sweden \\ ${ }^{3}$ University of Hail, College of Applied Medical Sciences, Department of Clinical Nutrition, Hail, Saudi Arabia
}

Received: November 29, 2015; Accepted: January 11, 2016; Published: January 13, 2016

*Corresponding author: Hadi Abd, Karolinska Institute, Department of Laboratory Medicine, Division of Clinical Microbiology F 82 and Karolinska University Hospital, Huddinge, SE-141 86 Stockholm, Sweden, Tel: +46-70-478-55-44; E-mail: hadi.abd@ki.se

\begin{abstract}
Intestinal parasitic infections affect billions of people worldwide. Low and middle incomes countries in the world are still using the wet mount method for the diagnosis in parasitology. The aims of our study were to determine the prevalence and distribution of intestinal parasitic infections among school students and to compare the wet mount method and the formalin-ethyl acetate concentration method for detecting intestinal parasites. Stool samples were collected from Malakal school students. 450 students aged 7 to 22 years in the Malakal Area in Upper Nile State, South Sudan. The samples were prepared by the wet mount and concentration methods and examined by microscopy. The overall prevalence of intestinal parasitic infections was $16.2 \%$ using the direct wet mount method and $18.6 \%$ by the formalin-ethyl concentration method. Both methods showed that Giardia intestinalis was the most predominant parasite followed by Hymenolepis nana, Entamoeba coli, Entamoeba histolytica/ E. dispar, Ascaris lumbricoides, Schistosoma mansoni and Trichuris trichiura. Double or multiple infection appeared in some cases as protozoa-protozoa or protozoa-helminths. Infections in males and primary level school students showed a higher prevalence than in females and those of secondary level. This study demonstrated that the formalin-ethyl acetate concentration method detects higher number of parasites than the direct wet mount method. G. intestinalis and H. nana are the most predominant parasites observed in school students in Malakal city. Formalin-ethyl acetate concentration method is recommended for the diagnosis of intestinal parasites.
\end{abstract}

Keywords: Intestinal parasites; School students; Microscopy methods

\section{Introduction}

Human intestinal parasites including helminths and protozoa are major causative agents of human diseases in most of the tropical countries. Helminths such as Ascaris lumbricoides [1], Hymenolepis nana [2] and Trichuris trichiura [3] infect billions of people, especially children, where protein energy malnutrition and anaemia are also prevalent, in addition access to medical care and educational opportunities are often limited.

Amoebiasis caused by protozoan Entamoeba histolytica in the low and middle income nations affected $50 \%$ of the general population causing more than 100000 deaths per year [4]. Giardia intestinalis, the protozoic causative agent of giardiasis, infects approximately 200 million people [2,5].

In this context, the population in South Sudan has been nearly completely evacuated over the course of the long civil war [6]. However, less information about parasitic infections in South Sudan is available and this paper tries to highlight the situation in the country.

Diagnosis of intestinal parasitic infections is routinely based on microscopy. Preparation of stool samples for microscopy performed by the direct wet mount method or the concentration methods (sedimentation and flotation). Sensitivity of the direct wet mount method was shown to be low [7] but, this method is still used in low and middle income countries.

The concentration technique has become a routine procedure in the ova and parasite examination and allows the detection of small numbers of organisms that may be missed by using only a direct wet mount [1]. The aims of this study were to determine the prevalence and distribution of intestinal parasitic infections among school students, and to compare between the wet mount method and the formalin-ethyl acetate concentration method in detecting intestinal parasites.

\section{Materials and Methods}

\section{Area of study, population and sample collection}

The study was conducted in Malakal area, upper Nile state in South Sudan during the period from August-November 2010. The state has an area of $77,773 \mathrm{~km}^{2}$ and an estimated population of approximately 1,400,000.

Malakal School was located in Malakal city that is the capital of the state. The total number of the students in the Malakal school was 450 including 236 boys and 214 girls, with age range 7-22 years. The number of students studying in primary (7-15 years) and secondary (16-22 year) levels was 200 and 250 respectively. 
Each student was given a labeled container for faecal sample collection. The students brought their samples back from home and delivered them to the medical team the same morning at school. Formalin was added to each sample and the samples transmitted to University of Medical Sciences and Technology, Khartoum, Sudan to be examined after 24 hour.

\section{Parasites identification and methods of examination}

The stool samples were prepared by the wet direct mount and the formalin-ethyl acetate concentration methods. Saline and iodine wet mounts of fresh unpreserved- and concentrated stool samples were examined microscopically for demonstrating trophozoites and cysts of protozoa and ova or larvae of helminths [1]. All the mounted area of each slide was screened by three well trained technologists before reporting negative results. Identification of parasites was based on microscopic morphology of the helminthic eggs / larvae and protozoic trophozoites / cysts. The microscopic morphology of the parasites was then compared with those in standard texts, literature and micrographs according to Centers of Disease Control and Prevention (CDC) standard methods for diagnosis of intestinal parasites [1].

\section{Ethical considerations}

The study protocol was reviewed and approved by the International Review Board (IRB 00008867) of the Sudan Medical and Scientific Research Institute (SUMASRI), University of Medical Sciences and Technology, Khartoum, Sudan. Written informed consent was obtained from all participants and mothers/ caretakers of children under 18 years old who participated in the study after explaining the purpose and objective of the study.

\section{Statistical analyses}

$\chi^{2}$ test was used for comparison between the two methods in detecting of intestinal parasites and their distribution according age and gender. A $p$ value of $\leq 0.05$ was considered statistically significant.

\section{Results}

A total of 450 students consisted of 236 (52\%) male and 214 (48\%) female, with age range 7-22 years. The number of students studying in primary (7-15 year) and secondary (16-22 year) levels were $200(44 \%)$ and 250 (56\%) respectively. The study aimed to estimate the prevalence and distribution of intestinal parasitic infections among school students utilizing the direct wet method and formalin-ethyl acetate concentration method.

\section{Prevalence of detected parasites by direct wet mount method}

Out of 450 students, 73 (16.2\%) were positive for parasites by the direct method. Giardia intestinalis, Entamoeba coli, Entamoeba histolytica/ E. dispar, Hymenolepis nana, Ascaris lumbricoides, Schistosoma mansoni and Trichuris trichiura were observed in $4.7 \%, 3.1 \%, 1.6 \%, 3.3 \%, 0.7 \%, 0.7 \%$ and $0.2 \%$ samples, respectively (Table 1).

Difference in number between protozoic and helminthic infections by this method was significant by $\chi^{2}$ test $(p<0.001)$.
Moreover, prevalence of infections with multiple protozoa was $0.7 \%$ E. coli and G. intestinalis, $0.4 \%$ E. coli and E. histolytica/ E. dispar, and $0.2 \%$ E. histolytica/E. dispar and G. intestinalis. The prevalence of multiple infections with both helminths and protozoa was $0.4 \% H$. nana and $G$. intestinalis, and $0.2 \% H$. nana and E. coli. The overall prevalence of alone protozoic and helminthic infections by direct method were $9 \%$ and $5 \%$ and that of multiple protozoic or protozoic/ helminthic infections were $1 \%$ and $1 \%$ respectively (Table 1 ).

\section{Prevalence of detected parasites by formalin-ethyl acetate concentration method}

Out of 450 students, 84 (18.6\%) were positive for parasites by the concentration method. Giardia intestinalis, Entamoeba coli, Entamoeba histolytica/E. dispar, Hymenolepis nana, Ascaris lumbricoides, Schistosoma mansoni and Trichuris trichiura were observed in $5.1 \%, 3.3 \%, 2.0 \%, 3.8 \%, 0.7 \%, 0.7 \%$ and $0.2 \%$ samples, respectively (Table 1). Difference in number between protozoic and helminthic infections by this method was highly significant by $\chi^{2}$ test $(p<0.0001)$.

Moreover, prevalence of infections with multiple protozoa was $1.1 \%$ E. coli and G. intestinalis, $0.7 \%$ E. coli and E. histolytica/ E. dispar, and $0.2 \%$ E. histolytica/ E. dispar and G. intestinalis. The prevalence of multiple infections with both helminths and protozoa was $0.7 \%$ H. nana and G. intestinalis, and $0.2 \% \mathrm{H}$. nana and E. coli (Table 1). The overall prevalence of alone protozoicand helminthic infections by concentration method were $10 \%$ and $5 \%$ and that of multiple protozoic or protozoic/ helminthic infections were $2 \%$ and $1 \%$. Comparison between number of protozoic and helminthic infections estimated by direct wet mount or formalin-ethyl acetate concentration method was very highly significant by $\chi^{2}$ test $(p<0.00001)$. However, both methods showed that the most predominant protozoic- and helminthic parasites in this study were G. intestinalis (Figure 1) and H. nana (Figure 2), respectively.

Table 1: Prevalence of intestinal parasites among school 450 students by direct mount and formalin-ethyl acetate concentration method.

\begin{tabular}{|l|l|l|}
\hline \multirow{2}{*}{ Parasites species } & $\begin{array}{l}\text { Prevalence } \\
\text { Direct wet } \\
\text { method }\end{array}$ & $\begin{array}{l}\text { Concentration } \\
\text { method }\end{array}$ \\
\hline Giardia intestinalis & $21(4.7 \%)$ & $23(5.1 \%)$ \\
\hline Entamoeba coli & $14(3.1 \%)$ & $15(3.3 \%)$ \\
\hline E. histolytica/E. dispar & $7(1.6 \%)$ & $9(2.0 \%)$ \\
\hline E. coli and G. intestinalis & $3(0.7 \%)$ & $5(1.1 \%)$ \\
\hline E. coli and E. histolytica/E. dispar & $2(0.4 \%)$ & $3(0.7 \%)$ \\
\hline $\begin{array}{l}\text { E. histolytica/E. dispar and G. } \\
\text { intestinalis }\end{array}$ & $1(0.2 \%)$ & $1(0.2 \%)$ \\
\hline Hymenolapis nana & $15(3.3 \%)$ & $17(3.8 \%)$ \\
\hline Ascaris lumbricoides & $3(0.7 \%)$ & $3(0.7 \%)$ \\
\hline Schistosoma mansoni & $3(0.7 \%)$ & $3(0.7 \%)$ \\
\hline Trichuris trichiura & $1(0.2 \%)$ & $1(0.2 \%)$ \\
\hline H. nana and G.intestinalis & $2(0.4 \%)$ & $3(0.7 \%)$ \\
\hline H. nana and E. coli & $1(0.2 \%)$ & $1(0.2 \%)$ \\
\hline Total & $73(16.2 \%)$ & $84(18.7 \%)$ \\
\hline
\end{tabular}




\section{Distribution of intestinal parasitic infections according age and gender}

Both direct wet mount and formalin-ethyl acetate concentration methods found that number of infected students decreased according to increased age, and the concentration method detected more infections in both age groups than the wet mount one (Table 2). Using the direct wet mount method, the frequency was $19 \%(47 / 248)$ in 7-15 years group, which decreased to $13 \%(26 / 202)$ in $16-22$ years group. The concentration method found that the frequency was 22\% (54/ $284)$ in 7-15 years group, which decreased to $15 \%$ (30/202) in 16-22 years group (Table 2). By the direct wet mount method, the frequency was $18 \%(42 / 236)$ in male group, which decreased to $14 \%$ (31/ 214) in female group. The concentration method found that the frequency was $20 \%(47 / 236)$ in male group, which decreased to $17 \%(37 / 214)$ in female group. Both direct and concentration method demonstrated significant differences in number of infected students between each age group and each

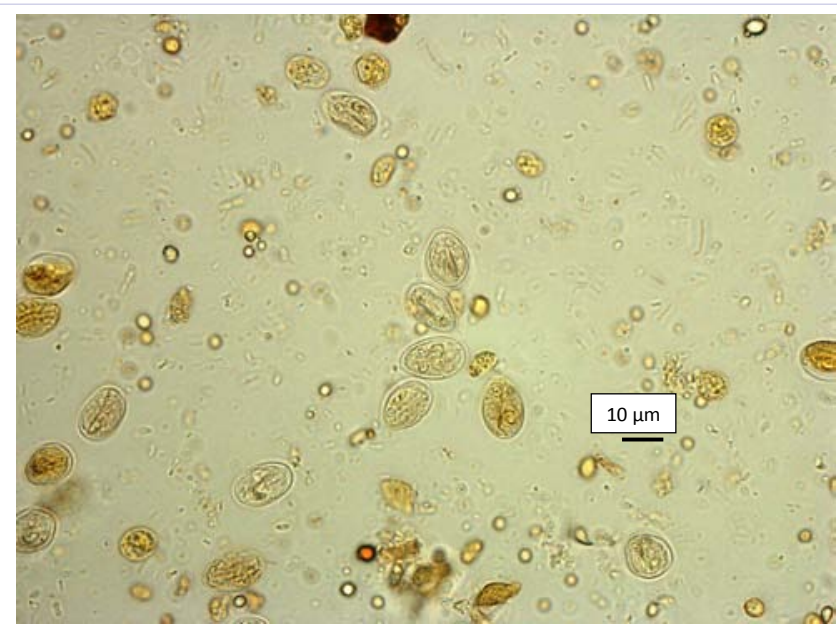

Figure 1: The figure demonstrates the protozoic parasite Giardia intestinalis cysts $1000 \mathrm{x}$.

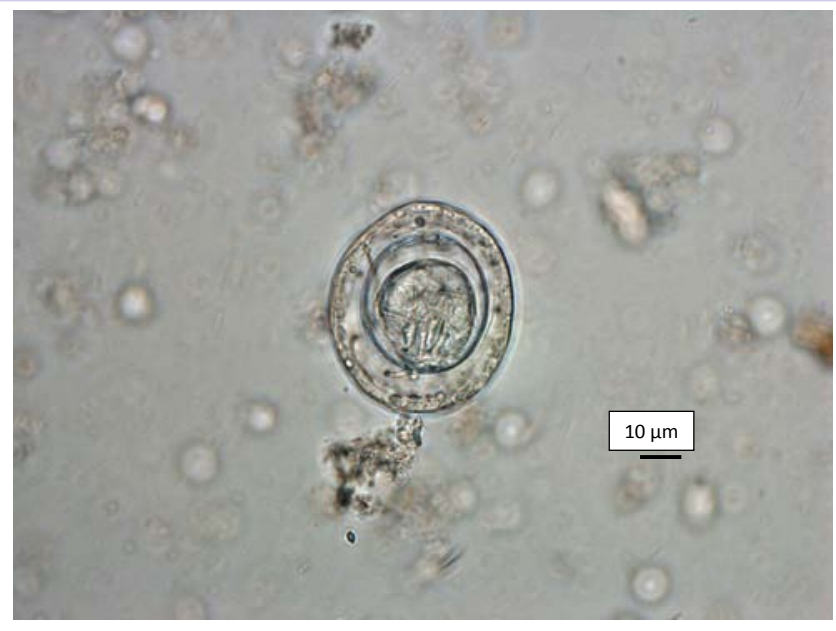

Figure 2: The figure demonstrates the helminthic parasite Hymenolepis nana egg $1000 \mathrm{x}$. gender group, $p$ of $\chi^{2}$ were less than 0.0001 (Table 2).

Moreover, comparison between concentration method and direct mount in detection of intestinal parasitic infections in same age group and same gender group showed that the concentration method found higher frequencies in 7-15 years group and in male group compared to the direct wet mount, $p$ of $\chi^{2}$ were less than 0.0001 (Table 2).

Generally, the formalin-ethyl acetate concentration method detected 84 positive samples compared to the direct mount method that detected only 73 positive samples indicating that the concentration method detected 11 positive samples more increasing the detection with $15 \%$ (11/ 73×100).

\section{Discussion}

Our current study conducted among 450 students at Malakal School in South Sudan found that the prevalence of parasitic infections was $18.6 \%$ and the detected parasites were G. intestinalis, H. nana, E. coli, E. histolytica/E. dispar, A. lumbricoides, $S$. mansoni and T. trichiura. The most frequently identified parasites in our study were $G$. intestinalis followed by H. nana. Karrar 1995 and Mamoun 2009 [8,9] in Sudan detected as our study $G$. intestinalis as a predominant parasite and other parasites E. histolytica/E. dispar, H. nana and T. trichiura showed lower frequencies than our result [8, 9]. However, in South Sudan E. coli and E. histolytica were the predominant protozoa and hookworms were the predominant helminths [10]. Despite that we detected $S$. mansoni in both methods used in our study we could not find eggs of hookworms, since methods to detect parasite eggs or larvae in stool samples lack high sensitivity, particularly when infection intensities are low. The most widely used method for the diagnosis of S. mansoni, hookworm, roundworm and whipworm in epidemiological surveys is the Kato-Katz technique. Recently, the FLOTAC technique has shown a higher sensitivity than the Kato-Katz method for the diagnosis of hookworm, roundworm and whipworm (11).

In Dohuk city northern Iraq, the frequency of $G$. intestinalis infection was 38.5\% [12]. In Sherborn town and nearby villages in Egypt, the most frequently protozoa were E. histolytica/E. dispar and $G$. intestinalis and the most prevalent helminths were H. nana and E. vermicularis [13]. In Ethiopia, the most predominant parasites were helminths; A. lumbricoides, $S$. mansoni, hookworms, H. nana, E. vermicularis and Strongyloides stercoralis compared to protozoa; G. intestinalis and E. histolytica/ dispar [14].

In our study, the most predominant parasites were protozoa followed by helminths as demonstrated by both wet mount and concentration methods. However, comparison between number of protozoan and helminthic infections estimated by the direct method or the concentration method was very highly significant by $\chi^{2}$ test $(p<0.00001)$. Moreover, both examination methods demonstrated a significant difference in number of infected students between age groups and gender groups as well ( $p$ of $\chi^{2}$ was $\left.<0.0001\right)$. However, the more infected groups were the males and the most infected group from the males was the 7-15 
Table 2: Distribution of intestinal parasitic infections among school students according to age group and gender by direct mount and formalin-ethyl acetate concentration method.

\begin{tabular}{|c|c|c|c|c|c|c|}
\hline Methods & Age/gender & Positive & Negative & Total & Frequency & $P$ of $\chi^{2}$ \\
\hline \multirow{5}{*}{ Direct mount } & $7-15$ year & 47 & 201 & 248 & $19 \%$ & \multirow{2}{*}{$<0.0001$} \\
\hline & 16-22 year & 26 & 176 & 202 & $13 \%$ & \\
\hline & & & & & & \\
\hline & Males & 42 & 194 & 236 & $18 \%$ & \multirow{2}{*}{$<0.0001$} \\
\hline & Females & 31 & 183 & 214 & $14 \%$ & \\
\hline \multirow{4}{*}{ Concentration } & 16-22 year & 30 & 172 & 202 & $15 \%$ & $<0.0001$ \\
\hline & & & & & & \\
\hline & Males & 47 & 189 & 236 & $20 \%$ & \multirow{2}{*}{$<0.0001$} \\
\hline & Females & 37 & 177 & 214 & $17 \%$ & \\
\hline
\end{tabular}

year group. These findings were similar to studies conducted in Turkey and in Sudan [9]. This gender and age group might be more exposed to possible risk factors of infection with soil transmitted helminths especially in regions having low economic and hygienic levels and bad habits of eating fruits without proper washing.

Multiple parasiticinfections were not reported by the previous studies $[8,9]$. Our study reported that helminths-protozoa multiple infections as $H$. nana and G. intestinalis or H. nana and E. coli were found but not helminths-helminths infections. AlSaeed found that 70 of 486 infected samples showed double or triple infections since $G$. intestinalis was combined with $H$. nana Blastocystis hominis, E. histolytica/E. dispar and Iodamoeba butschilii [12]. Moreover, multiple helminths-helminths or helminths-protozoa or protozoa-protozoa infections were reported by Abate et al., 2013 [14].

The wet mount method is widely used in Sudan. A previous study conducted by Mamoun et al., 2009 [9] in Sudan found a significant difference between stool examination by the direct wet mount preparation and the formalin-ethyl concentration in all samples from children in a rural area [9].Our result demonstrated that the formalin-ethyl acetate concentration method detected 84 positive samples compared to the direct mount method that detected only 73 positive samples indicating that the concentration method detected 11 positive samples more, which increased the detection with 15\% (11/ 73×100). In routine detection of parasites, microscopy is the standard method having high specificity and sensitivity especially detection of blood parasites (malaria), urinary tract parasites and intestinal parasites. Microscopy has less limitations regarding differentiation between Entamoeba species named E. histolytica and E. dispar and cannot differentiate between Cryptosporidium species. Nowadays, molecular biological methods such as Polymerase Chain Reaction (PCR) will differentiate between these species. However, these methodologies are expensive and not present in poor areas around the world. Depending on morphology of the parasites, microscopy is able to rapidly identify all phases of the different parasites found in the same sample (including cysts, trophozoites, ova and larvae) but PCR identifies just one parasite because of limitation of the
PCR primer. Even the available commercial panels PCR assay (RIDA ${ }^{\circledR}$ GENE Parasitic Stool Panel) [15] cannot detect more than four microorganisms of the diarrheal protozoa. More recently, direct smear was shown to have a sensitivity of $61 \%$ compared to formal ether concentration (92\%) and Kato Katz (60\%) for the detection of a variety of parasites including protozoa [7].

Our study, conducted among 450 students in Malakal School in South Sudan found that the prevalence of parasitic infection was $18.6 \%$. This high prevalence of parasitic infections in the unstable area and the threat of cholera and other waterborne diseases [16] warrant urgent international support to build South Sudan.

\section{Conclusion}

G. intestinalis and H. nana are the most predominant parasites observed in school children in Malakal city. Formalin-ethyl acetate concentration method is recommended for the diagnosis of intestinal parasites.

\section{References}

1. Centers for Disease Control \& Prevention (CDC), Laboratory Identification of Parasites of Public Health Concern (DPDx), Atlanta,USA. http://www.cdc.gov/dpdx/diagnosticProcedures/ stool/index.html.

2. Kucik CJ, Martin GL, Sortor BV. Common intestinal parasites. Am Fam Physician. 2004;69(5):1161-8.

3. Stephenson LS, Holland CV, Cooper ES. The public health significance of Trichuris trichiura. Parasitology. 2000;121 Suppl:S73-95.

4. Choubisa SL, Jaroli VJ, Choubisa P, Mogra N. Intestinal parasitic infection in Bhil tribe of Rajasthan, India. J Parasit Dis. 2012;36(2):1438. doi: 10.1007/s12639-012-0151-y.

5. Minenoa T, Avery MA. Giardiasis: recent progress in chemotherapy and drug development. Curr Pharm Des. 2003;9(11):841-55.

6. International R. 2010. http://www.ri.org/where_we_work/country. php?ID=27.

7. Hailu T, Abera B. Performance evaluation of direct saline stool microscopy, Formol ether concentration and Kato Katz diagnostic methods for intestinal parasitosis in the absence of gold standard methods. Trop Doct. 2015;45(3):178-82. doi: 10.1177/0049475515581127. 
8. Karrar ZA, Rahim FA. Prevalence and risk factors of parasitic infections among under-five Sudanese children: a community based study. East Afr Med J. 1995;72(2):103-9.

9. Mamoun MM, Abubakr IA, Salah ET. Frequency of intestinal parasitic infections among displaced children in Kassala Town. Khartoum Medical Journal. 2009;2(1):175 - 177.

10. Magambo JK, Zeyhle E, Wachira TM. Prevalence of intestinal parasites among children in Southern Sudan. East Afr Med J. 1998;75(5):28890.

11. Glinz D, Silué KD, Knopp S, Lohourignon LK, Yao KP, Steinmann P, et al. Comparing diagnostic accuracy of Kato-Katz, Koga agar plate, ether-concentration, and FLOTAC for Schistosoma mansoni and soiltransmitted helminths. PLoS Negl Trop Dis. 2010;4(7):e754. doi: 10.1371/journal.pntd.0000754.

12. Al-Saeed AT, Issa SH. Frequency of Giardia lamblia among children in
Dohuk, northern Iraq. East Mediterr Health J. 2006;12(5):555-61.

13. Hanafi FZ, Abdel-Magied SA, Abdel-Wahab FM. Prevalence of parasitic diseases among primary school children in Sherbeen area, Dakahlia, Egypt. J Egypt Soc Parasitol. 1985;15(2):543-52.

14. Abate A, KibretB, Bekalu E, Abera S, Teklu T, Yalew A et.al. CrossSectional Study on the Prevalence of Intestinal Parasites and Associated Risk Factors in Teda Health Centre, Northwest Ethiopia. 2013. ISRN Parasitology, Article ID 757451, 5 pages, http://dx.doi. org/10.5402/2013/757451

15. Andreas S, Heidrun VT, Egbert T. 2013. http://pascv.ivdnews.net/ public/show_abstract/1373

16. JOM South Sudan, Situation Report. 2014. www.iom.int/files/live/ sites/iom/files/Country/docs/IOM-South-Sudan-Situation-Report26-April-2014.pdf. 